\title{
Optimal Channel Shortening Equalization for MIMO ISI Channels
}

\author{
Raman Venkataramani \\ Seagate Technology \\ 1251 Waterfront Place, Pittsburgh, PA 15222 \\ Email: ramanv@ieee.org
}

\author{
Sundararajan Sankaranarayanan \\ Seagate Technology \\ 1251 Waterfront Place, Pittsburgh, PA 15222 \\ Email: sundararajan.sankaranarayanan@seagate.com
}

\begin{abstract}
We consider the problem of channel shortening equalization to perform reduced complexity detection for MIMO channels with intersymbol interference and additive Gaussian noise. the problem is to find a MIMO equalizer and a partial response MIMO target filter such that the combination produces the best detection performance. We show the existence of an infinite family of optimal equalizers and targets that satisfy the a posteriori equivalence condition. Furthermore, these solutions are intimately related to MIMO decision feedback equalizers designed with the "monic determinant" constraint on the feedback filter. These results generalize some recent results on channel shortening based on a posteriori equivalence to the MIMO setting.
\end{abstract}

\section{INTRODUCTION}

Multiple input multiple output (MIMO) communication systems have been an emerging field in recent years. For example, in wireless communications, the use of multiple antennas for transmission and reception is desirable because in a Rayleigh flat fading environment the capacity grows linearly with the minimum of the number of transmitter and receiver antennas [1]. Another example is multi-head multi-track recording [2], [3] where there is intertrack interference. These and many other communications systems can be modeled as MIMO intersymbol interference (ISI) channels.

Orthogonal frequency division multiplexing (OFDM) solves the ISI problem for wireless channels. However it does notapply to all channels like the magnetic recording channel for which the channel input or magnetization is constrained to \pm 1 . In this paper, we are primarily concerned with optimal channel shortening and reduced complexity detection for MIMO ISI channels. In principle we can adapt the Viterbi algorithm to perform maximum-likelihood (ML) or maximum a posteriori (MAP) detection of the input sequence in the MIMO setting. However, the complexity grows like $2^{N(I+1)}$ for an $N \times N$ MIMO system with channel memory $I$ due to ISI. The purpose of this work is to identify the set of MIMO equalizers and targets that yield the best performance with respect to ML and MAP-based detection for the equalized channel. Generalizing the results in [4], [5] to the MIMO case, we show the existence of a family of equalizers and targets satisfying the a posteriori equivalence or "APP matching". These solutions are intimately related to the minimum mean-square (MMSE) decision feedback equalizers (DFE) designed with a monic determinant constraint on the feedback filter. These ideas are adapted to to design optimal FIR targets and equalizers. We review our notation and some basic results on matrix spectral factorization before addressing the problem of channel shortening equalization for MIMO systems.

\section{A. Definitions and Notation}

Henceforth, we refer to a sequence of matrices or vectors simply as a sequence. Let $\boldsymbol{a}$ denote a discrete-time sequence $\{\boldsymbol{a}[n]: n \in \mathbb{Z}\}$. The $z$-transform of $\boldsymbol{a}$ is defined as

$$
\boldsymbol{A}(z)=\sum_{n} \boldsymbol{a}[n] z^{n}
$$

If $\boldsymbol{a}$ has finite energy then its discrete-time Fourier transform $\mathcal{F}\{\boldsymbol{a}\}$ is the restriction of the $\boldsymbol{A}(z)$ on the unit circle and is denoted by $\boldsymbol{A}(\omega)$ for convenience. The convolution of two sequences $\boldsymbol{a}$ and $\boldsymbol{b}$ is denoted by $\boldsymbol{c}=\boldsymbol{a} \star \boldsymbol{b}$ where

$$
\boldsymbol{c}[n]=\sum_{m} \boldsymbol{a}[m] \boldsymbol{b}[n-m]
$$

Let $\boldsymbol{\delta}$ denote the discrete delta function: $\delta[n]=\mathbf{0}_{N \times N}$ for $n \neq 0$ and $\delta[0]=\boldsymbol{I}_{N \times N}$. Define the inner product between two sequences $\boldsymbol{a}$ and $\boldsymbol{b}$ as

$$
\langle\boldsymbol{a}, \boldsymbol{b}\rangle=\operatorname{tr} \sum_{n} \boldsymbol{a}^{*}[n] \boldsymbol{b}[n]=\frac{1}{2 \pi} \int_{-\pi}^{\pi} \boldsymbol{A}^{*}(\omega) \boldsymbol{B}(\omega) d \omega
$$

where $\operatorname{tr}(\cdot)$ is the trace and $*$ denotes the Hermitian (adjoint) of a matrix. Thus, the norm of $\boldsymbol{a}$ is $\|\boldsymbol{a}\|=\langle\boldsymbol{a}, \boldsymbol{a}\rangle^{1 / 2}$. Given a sequence $\boldsymbol{a}$, let $\ddot{\boldsymbol{a}}$ be obtained by time-reversal and conjugation of $\boldsymbol{a}$, i.e., $\ddot{\boldsymbol{a}}[n]=\boldsymbol{a}^{*}[-n]$. The Fourier transform of $\ddot{\boldsymbol{a}}$ is $\boldsymbol{A}^{*}(\omega)$. Thus, we readily obtain the following identity:

$$
\langle a \star b, c\rangle=\langle\boldsymbol{b}, \ddot{a} \star c .\rangle
$$

The cross-correlation function of a real or circularly symmetric complex stationary random processes $\boldsymbol{x}$ and $\boldsymbol{y}$ is defined as $\boldsymbol{r}_{x y}[n]=\zeta^{-1} \mathrm{E}\left(\boldsymbol{x}[m+n] \boldsymbol{y}^{*}[m]\right)$ where $\mathrm{E}(\cdot)$ denotes expectation and $\zeta$ is the number of real dimensions per sample, i.e., $\zeta=1$ for real processes and $\zeta=2$ for complex ones. The autocorrelation of $\boldsymbol{x}$ is obtained by setting $\boldsymbol{y}=\boldsymbol{x}$. The power spectral density of $\boldsymbol{x}$ is $\boldsymbol{S}_{x}(\omega)=\mathcal{F}\left\{\boldsymbol{r}_{x x}\right\}$. We write $\boldsymbol{x} \perp \boldsymbol{y}$ if $\boldsymbol{r}_{x y}=0$. All complex-valued processes are assumed to be circularly symmetric. 


\section{B. Spectral Factorization}

We now review the notion of minimum-phase decomposition for matrix polynomials [6], [7].

Definition 1. Suppose that $\{\boldsymbol{a}[m]: m \geq 0\}$ is a causal sequence of $N \times N$ matrices satisfying the Paley-Wiener condition:

$$
\int_{-\pi}^{\pi} \log |\operatorname{det} \boldsymbol{A}(\omega)| d \omega>-\infty .
$$

Then $\boldsymbol{A}(z)$ is called minimum-phase if $\operatorname{det} \boldsymbol{A}(z)$ is minimumphase and both $\boldsymbol{A}(z)$ and its inverse are analytic for $|z| \geq$ 1. Additionally, we term $\boldsymbol{A}(z)$ as canonical if $\boldsymbol{a}[0]$ is lowertriangular with nonnegative diagonal entries.

Informally, we say that $\boldsymbol{A}(\omega)$ is minimum-phase or canonical if its analytic extension $\boldsymbol{A}(z)$ is minimum-phase or canonical. The following result describes minimum-phase spectral factorization for matrices [6].

Proposition 1. Let $\boldsymbol{Q}(\omega)$ be an $N \times N$ non-negative definite Hermitian matrix satisfying the regularity condition (2). Then, $\boldsymbol{Q}(\omega)$ can be uniquely factorized as

$$
\boldsymbol{Q}(\omega)=\boldsymbol{G}_{\circ}^{*}(\omega) \boldsymbol{G}_{\circ}(\omega)
$$

where $\boldsymbol{G}_{\circ}(\omega)$ is a canonical minimum-phase factor.

Without the lower-triangular restriction, $\boldsymbol{G}(\omega)=\boldsymbol{U} \boldsymbol{G}_{\circ}(\omega)$ would be a valid minimum-phase spectral factor for any unitart $\boldsymbol{U}$. We are simply using the Cholesky factorization to generate a unique spectral factor. The canonical decomposition (3) is a generalization of the minimum-phase decomposition for polynomials and the Cholesky factorization for matrices. We could equally well have chosen an upper-triangular $\boldsymbol{g}_{\circ}[0]$, or more generally forced $\boldsymbol{P} \boldsymbol{g}_{\circ}[0] \boldsymbol{P}^{-1}$ to be a lower-triangular for some permutation matrix $\boldsymbol{P}$. There are several algorithms to compute the matrix spectral factors including the Bauer factorization algorithm [7].

\section{Channel Model}

Consider a discrete-time real or complex-valued linear time invariant system with $N$ inputs and $M \geq N$ outputs

$$
y=h \star x+w
$$

where $\boldsymbol{h}=\{\boldsymbol{h}[m]: m \in \mathbb{Z}\}$ is the channel response, $\boldsymbol{x}=\{\boldsymbol{x}[m]: m \in \mathbb{Z}\}$ is the input, and $\boldsymbol{w}=\{\boldsymbol{w}[m]: m \in \mathbb{Z}\}$ is additive white Gaussian noise with power spectral density $\boldsymbol{S}_{w}(\omega)$. The sizes of $\boldsymbol{x}[m], \boldsymbol{w}[m]$ and $\boldsymbol{h}[m]$ are $N \times 1$, $M \times 1$, and $M \times N$ respectively. Assume that $\boldsymbol{h}$ has finite energy but is possibly non-causal and IIR. The channel model (4) is a MIMO ISI channel and describes a variety of real communication systems such as multiple antenna wireless communications over ISI channels or multi-track magnetic recording.

Let the input power spectral density be $\boldsymbol{S}_{x}(\omega)$. As a special case we also shall consider independent and identically distributed (IID) inputs with $\boldsymbol{S}_{x}(\omega)=\boldsymbol{I}$. An example for the input symbol set is the $Q$-phase PSK constellation,

$$
\mathcal{C}=\left\{\sqrt{2} e^{j 2 \pi q / Q}: q=0, \ldots, Q-1\right\}
$$

in the complex case or the BPSK (bipolar binary) constellation $\mathcal{C}=\{-1,+1\}$ in the real case.

Suppose that the MIMO ISI channel (4) has a noise power spectral density (PSD) of $\boldsymbol{S}_{w}(\omega)$. The whitened matched filter channel can be expressed as

$$
\overline{\boldsymbol{y}}=\boldsymbol{k} * \boldsymbol{y}=\overline{\boldsymbol{h}} \star \boldsymbol{x}+\overline{\boldsymbol{w}}
$$

where $\overline{\boldsymbol{w}}=\boldsymbol{k} \star \boldsymbol{w}$ and $\boldsymbol{k}$ is an $N \times M$ noise-whitening filter for which $\boldsymbol{S}_{\bar{w}}(\omega)=\boldsymbol{K}(\omega) \boldsymbol{S}_{w}(\omega) \boldsymbol{K}^{*}(\omega)=\boldsymbol{I}$. It is readily shown that $\overline{\boldsymbol{H}}(\omega)=\boldsymbol{K}(\omega) \boldsymbol{H}(\omega)$ and $\overline{\boldsymbol{H}}^{*}(\omega) \overline{\boldsymbol{H}}(\omega)=$ $\boldsymbol{H}^{*}(\omega) \boldsymbol{S}_{w}^{-1}(\omega) \boldsymbol{H}(\omega)$. We may assume without loss of generality that $\bar{h}$ is a canonical spectral factor. In this case we call the resulting channel the canonical noise whitened channel.

\section{Optimal Detection}

Consider the whitened noise channel (5). with $\overline{\boldsymbol{h}}=\boldsymbol{k} \star \boldsymbol{h}$ is in its canonical form and $\overline{\boldsymbol{w}}=\boldsymbol{k} \star \boldsymbol{w}$ is white noise. This is a convenient choice because it ensures that the ISI is causal. In principle the channel response $\boldsymbol{h}$ and noise statistics $\boldsymbol{r}_{w w}$ can be estimated through training as described below. Since the channel is noise whitened, we have

$$
P(\overline{\boldsymbol{y}} \mid \boldsymbol{x}) \propto \exp \left(-\frac{\|\overline{\boldsymbol{y}}-\overline{\boldsymbol{h}} \star \boldsymbol{x}\|^{2}}{2 \sigma_{\bar{w}}^{2}}\right) .
$$

Using Bayes' rule we express the a posteriori probability (APP) as

$$
\begin{aligned}
P(\boldsymbol{x} \mid \boldsymbol{y}) & =P(\boldsymbol{x} \mid \overline{\boldsymbol{y}})=P(\overline{\boldsymbol{y}} \mid \boldsymbol{x}) P(\boldsymbol{x}) / P(\overline{\boldsymbol{y}}) \\
& \propto P(\boldsymbol{x}) \exp \left(-\frac{\|\overline{\boldsymbol{y}}-\overline{\boldsymbol{h}} \star \boldsymbol{x}\|^{2}}{2 \sigma_{\bar{w}}^{2}}\right)
\end{aligned}
$$

where the proportionality constants above and henceforth are always independent of $\boldsymbol{x}$. The maximum a posteriori (MAP) sequence detector computes

$$
\hat{\boldsymbol{x}}:=\arg \max _{\boldsymbol{x}} P(\boldsymbol{x} \mid \overline{\boldsymbol{y}})
$$

which reduces to the maximum-likelihood (ML) detector if all input sequences are equally probable

$$
\hat{\boldsymbol{x}}:=\arg \min _{\boldsymbol{x}}\|\overline{\boldsymbol{y}}-\overline{\boldsymbol{h}} \star \boldsymbol{x}\|^{2} .
$$

The detector computes $\hat{\boldsymbol{x}}$ using the Viterbi algorithm. Likewise, the BCJR algorithm may be used to compute the symbol-wise APPs. The complexity of these detectors grows exponentially with the length of the whitened channel response $\overline{\boldsymbol{h}}[m]$. 


\section{Reduced Complexity Detection}

\section{A. Linear Equalization}

We briefly review standard techniques for equalization and target design for the MIMO ISI channel (4). Equalization is done to shorten the channel response to match a pre-specified target response reduce sequence detection complexity.

Let $\boldsymbol{f}$ and $\boldsymbol{g}$ denote the equalizer and target filters respectively. The output of the equalized channel (Fig. 1) is

$$
z=f \star y=l \star x+u
$$

where $\boldsymbol{l}=\boldsymbol{f} \star \boldsymbol{h}$ is the equalized channel and $\boldsymbol{u}=\boldsymbol{f} \star \boldsymbol{w}$ is the equalized noise with $\boldsymbol{S}_{u}(\omega)=\boldsymbol{F}(\omega) \boldsymbol{S}_{w}(\omega) \boldsymbol{F}^{*}(\omega)$. As in [4], [5] let us define the hypothetical target channel with output $\tilde{z}$, illustrated in Fig. 2, as

$$
\tilde{\boldsymbol{z}}=\boldsymbol{g} \star \boldsymbol{x}+\boldsymbol{v}
$$

where $\boldsymbol{v}$ is additive white Gaussian noise (AWGN) with $\boldsymbol{S}_{v}(\omega)=\sigma_{v}^{2} \boldsymbol{I}$. The tilde is used to differentiate between the output of (hypothetical) target channel from that of the equalized sequence. Thus,

$$
\tilde{P}(\tilde{\boldsymbol{z}} \mid \boldsymbol{x}) \propto \exp \left(-\frac{\|\tilde{\boldsymbol{z}}-\boldsymbol{g} \star \boldsymbol{x}\|^{2}}{2 \sigma_{v}^{2}}\right)
$$

denotes the channel probability function by related to the target channel. Traditionally, the equalizer and target are designed to make equalized channel response $\boldsymbol{l}$ close to target $\boldsymbol{g}$, while keeping the noise white. The detector treats the equalized channel and target channel as being equivalent. Thus, instead of (8) the reduced complexity ML sequence detector computes

$$
\hat{\boldsymbol{x}}:=\arg \min _{\boldsymbol{x}}\|\boldsymbol{z}-\boldsymbol{g} \star \boldsymbol{x}\|^{2} .
$$

More generally, MAP based detectors use

$$
\tilde{P}(\boldsymbol{x} \mid \boldsymbol{z}) \propto P(\boldsymbol{x}) \tilde{P}(\boldsymbol{z} \mid \boldsymbol{x}) \propto \exp \left(-\frac{\|\boldsymbol{z}-\boldsymbol{g} \star \boldsymbol{x}\|^{2}}{2 \sigma_{v}^{2}}\right)
$$

as the APP function. Note that the above quantity is the APP of $\boldsymbol{x}$ for the target channel computed with $\boldsymbol{z}$ rather that $\tilde{\boldsymbol{z}}$ even though $z$ may not be a typical output of the target channel. The reduced complexity approach could be suboptimal compared to the detectors (7) and (8) based on the actual channel response.

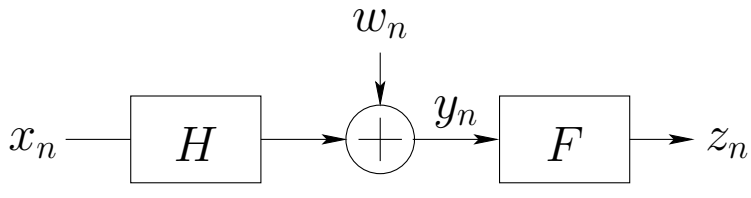

Fig. 1. Original channel and equalizer.

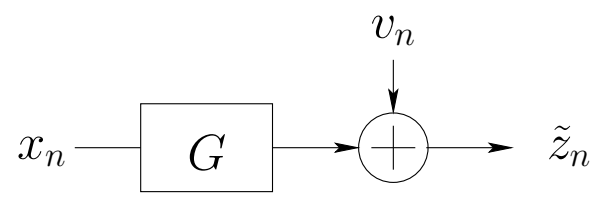

Fig. 2. Target channel.

Zero Forcing Equalization: A zero forcing equalizer (ZFE) $f$ is such that the equalized channel response equals the target response, i.e., $\boldsymbol{l}=\boldsymbol{g}$. Thus, in the frequency domain, the equalizer is given by $\boldsymbol{F}(\omega)=\boldsymbol{G}(\omega) \boldsymbol{H}^{-1}(\omega)$. An undesirable problem with zero-forcing equalization is that when the smallest singular value of channel response $\boldsymbol{H}(\omega)$ has a null or attains very small values, the equalized noise is highly colored and has large variance. The ZFE is rarely used for this reason.

MMSE Equalization: A commonly used equalizer is the minimum mean square error (MMSE) equalizer that minimizes the variance of the equalization error $e:=g \star x-f \star y$ :

$$
\mathcal{E}:=\mathrm{E}\|\boldsymbol{e}[n]\|^{2}=\frac{1}{2 \pi} \operatorname{tr} \int_{-\pi}^{\pi} \boldsymbol{S}_{e}(\omega) d \omega .
$$

Using $\boldsymbol{e} \perp \boldsymbol{y}$, and $\boldsymbol{S}_{x}=\boldsymbol{I}$ it is readily verified that

$$
\boldsymbol{F}(\omega)=\boldsymbol{G}(\omega) \boldsymbol{H}^{*}(\omega)\left(\boldsymbol{H}(\omega) \boldsymbol{H}^{*}(\omega)+\boldsymbol{S}_{w}(\omega)\right)^{-1}
$$

and the power spectral density of the estimation error is

$$
\begin{aligned}
\boldsymbol{S}_{e}(\omega) & =\boldsymbol{G}(\omega) \boldsymbol{Q}^{-1}(\omega) \boldsymbol{G}^{*}(\omega) \\
\boldsymbol{Q}(\omega) & =\boldsymbol{I}+\boldsymbol{H}^{*}(\omega) \boldsymbol{S}_{w}^{-1}(\omega) \boldsymbol{H}(\omega) .
\end{aligned}
$$

For scalar ISI channels, the MMSE decision feedback equalizer (DFE) filters is derived by minimizing the equalizer error variance subject to the monic constraint on the target [8], [9] where the strictly causal part of the target functions as the feedback filter. In the MIMO case, the standard approach has been to minimize the trace of the MMSE equalization error covariance subject to energy or identity tap constraints [10], [11] or a monic lower-triangular constraint [12] on the feedback filter. However we propose the "monic determinant" constraint on the MIMO target filter which is a natural generalization of the (scalar) monic constraint to the MIMO setting, as shown later.

\section{Proposition 2. The solution to the minimization of}

$$
\mathcal{E}=\frac{1}{2 \pi} \operatorname{tr} \int_{-\pi}^{\pi} \boldsymbol{G}(\omega) \boldsymbol{Q}^{-1}(\omega) \boldsymbol{G}^{*}(\omega) d \omega
$$

over all causal targets such that $\operatorname{det} \boldsymbol{g}[0]=1$ is the minimumphase spectral factor of $\boldsymbol{G}^{*}(\omega) \boldsymbol{G}(\omega)=\lambda \boldsymbol{Q}(\omega)$ where $\lambda$ is chosen to satisfy $\operatorname{det} \boldsymbol{g}[0]=1$.

In particular the optimal target may be taken as the canonical spectral factor. The proof of Proposition 2 is omitted for brevity. Thus, the the MMSE DFE target designed with the monic determinant constraint is the canonical spectral factor of

$$
\boldsymbol{G}^{*}(\omega) \boldsymbol{G}(\omega)=\lambda\left(\boldsymbol{I}+\boldsymbol{H}^{*}(\omega) \boldsymbol{S}_{w}^{-1}(\omega) \boldsymbol{H}(\omega)\right)
$$


and the resulting equalization error is white with $\boldsymbol{S}_{e}(\omega)=\lambda \boldsymbol{I}$.

The strictly causal part of $\{\boldsymbol{g}[k]: k>0\}$ constitutes the feedback filter in the DFE structure. In this paper we do not actually use decision feedback to perform detection, because the method leads to error propagation and is generally suboptimal. However, the IIR MMSE DFE filters derived above can be used as our equalizer and target filters for reduced complexity Viterbi detection. In the next section we shall see that the above solution enjoys certain optimality properties in the context of sequence detection.

\section{B. A Posteriori Equivalence}

In [4], [5] we introduced the notion of a posteriori equivalence (APE) between the equalized and target channels and showed that there exists a family of equalizers-target pairs for which there is no loss in detection performance treating the equalized and target channel as equivalent. The following theorem is a generalization of those results to the MIMO channel.

Theorem 1. Suppose that all the input sequences in the message codebook have equal energy and the equalizer and the target channel are chosen such that

$$
\begin{aligned}
\boldsymbol{F}(\omega) & =\boldsymbol{G}(\omega) \boldsymbol{H}^{*}(\omega)\left(\boldsymbol{H}(\omega) \boldsymbol{H}^{*}(\omega)+\beta \boldsymbol{S}_{w}(\omega)\right)^{-1} \\
\boldsymbol{G}^{*}(\omega) \boldsymbol{G}(\omega) & =\alpha\left(\boldsymbol{H}^{*}(\omega) \boldsymbol{S}_{w}^{-1}(\omega) \boldsymbol{H}(\omega)+\beta \boldsymbol{I}\right) \\
\sigma_{v}^{2} & =\alpha \sigma_{\bar{w}}^{2}
\end{aligned}
$$

for some $\alpha>0$ and $\beta \in \mathbb{R}$ that produces a valid $\boldsymbol{G}(\omega)$, then $P(\boldsymbol{x} \mid \overline{\boldsymbol{y}})=\tilde{P}(\boldsymbol{x} \mid \boldsymbol{z})$. Consequently, (12) produces the correct $A P P$ of $x$. In particular,

$$
\hat{\boldsymbol{x}}=\arg \min _{\boldsymbol{x}}\|\overline{\boldsymbol{y}}-\overline{\boldsymbol{h}} \star \boldsymbol{x}\|^{2} \equiv \arg \min _{\boldsymbol{x}}\|\boldsymbol{z}-\boldsymbol{g} \star \boldsymbol{x}\|^{2}
$$

i.e., (11) produces the optimal ML estimate of the input sequence.

Proof: It is easily verified that the hypotheses imply that

$$
\boldsymbol{G}^{*}(\omega) \boldsymbol{G}(\omega)=\alpha\left(\overline{\boldsymbol{H}}^{*}(\omega) \overline{\boldsymbol{H}}(\omega)+\beta \boldsymbol{I}\right)
$$

where $\overline{\boldsymbol{h}}$ is the response of the canonical noise-whitened channel (5) with the whitening filter $\boldsymbol{k}$. Omitting a few steps, we can show that

$$
\boldsymbol{G}^{*}(\omega) \boldsymbol{F}(\omega)=\alpha \boldsymbol{H}^{*}(\omega) \boldsymbol{S}_{w}^{-1}(\omega)=\alpha \overline{\boldsymbol{H}}^{*}(\omega) \boldsymbol{K}(\omega) .
$$

In the time domain, these conditions imply that $\boldsymbol{s}:=\ddot{\boldsymbol{g}} \star \boldsymbol{g}-$ $\alpha \ddot{\overline{\boldsymbol{h}}} \star \overline{\boldsymbol{h}}=\alpha \beta \boldsymbol{\delta}$ and $\ddot{\boldsymbol{g}} \star \boldsymbol{f}=\alpha \ddot{\overline{\boldsymbol{h}}}$. Using these conditions and the identity (1), it is easily verified that

$$
\begin{aligned}
\|\boldsymbol{z}-\boldsymbol{g} \star \boldsymbol{x}\|^{2}-\|\boldsymbol{z}\|^{2} & =\langle\boldsymbol{x}, \boldsymbol{s} \star \boldsymbol{x}\rangle+\alpha\left(\|\overline{\boldsymbol{y}}-\overline{\boldsymbol{h}} \star \boldsymbol{x}\|^{2}-\|\overline{\boldsymbol{y}}\|\right)^{2} \\
& =\alpha \beta\|\boldsymbol{x}\|^{2}+\alpha\left(\|\overline{\boldsymbol{y}}-\overline{\boldsymbol{h}} \star \boldsymbol{x}\|^{2}-\|\overline{\boldsymbol{y}}\|\right)^{2} .
\end{aligned}
$$

Since all input sequences have equal energy we readily obtain (18) because $\boldsymbol{z}$ and $\overline{\boldsymbol{y}}$ are constants as far as the above minimization is concerned. Furthermore, by comparing (6) and (12) for the above choice of $\sigma_{v}^{2}$ we have $P(\boldsymbol{x} \mid \overline{\boldsymbol{y}}) \propto \tilde{P}(\boldsymbol{x} \mid \boldsymbol{z})$. Obviously the constant of proportionality must be unity.

The optimal target in (19) can be computed using the canonical spectral factorization of the right-hand side of (19).
The interpretation of Theorem 1 is that the equalized channel and the hypothetical target channel are a posteriori equivalent in the sense that they generate the same APP for the input $\boldsymbol{x}$. Furthermore, there is an infinite family of such solutions, parameterized by $\beta$. Thus, we can design any MAP based detector for the target channel, and it would be simultaneously optimal for the equalized channel. This fact was previously pointed out for scalar ISI channels in [4], [5]. We reiterate that the two channels are not equivalent in any other sense. For example they could have different forward channel laws: $P(\boldsymbol{z} \mid \boldsymbol{x}) \neq \tilde{P}(\boldsymbol{z} \mid \boldsymbol{x})$. Similarly, the sequence $\boldsymbol{z}$ may not be a typical output of the target channel: $P(\boldsymbol{z}) \neq \tilde{P}(\boldsymbol{z})$.

Theorem 1 is applicable to input sequences that have equal symbol energies, including symbols from the (complex) $Q$ PSK or (real) BPSK constellations. The result can also be adapted to apply to inputs with unequal symbol energies if we carefully fake the input prior distribution of the target channel as described in [4].

Relation to MMSE DFE Solution: The family of solutions in Theorem 1 are in fact closely related to the MMSE DFE for the MIMO ISI channel. The equalizer and target in Theorem 1 are precisely equal to the MMSE equalizer (14) and the DFE prediction filter (17) if $\beta=1$ and $\alpha=\lambda$. Note that $\alpha$ is a scaling factor but $\beta$ is a free parameter that generates an infinite family of optimal solutions. For an arbitrary $\beta>0$ the above quantities would be the MMSE DFE designed for an additive noise with power spectral density $\beta \boldsymbol{S}_{w}(\omega)$. This result can be interpreted as the MIMO version of the main result in [4], [5]. Thus, the monic determinant constraint on the target filter is special because it guarantees APE or "APP matching" for MIMO ISI channels. In general, other target constraints including the identity tap, energy, or monic lower-triangular constraints do not enjoy this property. If we had imposed a constraint on $\boldsymbol{g}[0]$ such as the monic lowertriangular constraint or $\boldsymbol{g}[0]=\boldsymbol{I}$, then $\boldsymbol{S}_{e}(\omega)$ would be independent of $\omega$ but not necessarily a scaled identity matrix. In this case APE is achievable if $v$ in target channel is treated as colored noise with covariance $\boldsymbol{S}_{e}(\omega)$. However, this would complicate the Viterbi metric and the cleaner approach is to use the $\operatorname{det} \boldsymbol{g}[0]=\boldsymbol{I}$ constraint which yields $\boldsymbol{S}_{e}(\omega)=\lambda \boldsymbol{I}$.

\section{FIR MIMO Equalizer and Target Design}

The analytical expressions for the optimal MMSE equalizer and target derived so far are IIR in general. We now consider the design of generalized partial response (GPR) targets for the MIMO channel where we seek the best target of a certain length. Using the MMSE equalization error as our cost function. Thus, we minimize the variance of (13) over all $f$ and causal $\boldsymbol{g}$ subject to the monic determinant constraint

$$
\operatorname{det} \boldsymbol{g}[0]=1 \Longleftrightarrow \operatorname{deg} \boldsymbol{G}(z) \text { is monic. }
$$

which is the natural constraint in the IIR case since it manifests a posteriori equivalence of the equalized an target channel. For simplicity, we do not consider the delay optimization problem below [11]. 
Let the equalizer and target be FIR filters $\boldsymbol{f}=\{\boldsymbol{f}[k]$ : $-K \leq k \leq K\}$ and $\boldsymbol{g}=\{\boldsymbol{g}[k]: 0 \leq k \leq L\}$ respectively. First consider the MMSE equalizer design for a fixed target. We seek the equalizer $f$ that minimizes (13). The solution is obtained by solving $\mathrm{E}\left(\boldsymbol{e}[n] \boldsymbol{y}^{*}[n-l]\right)=0$ for $-K \leq l \leq K$ which yields $\gamma \boldsymbol{R}_{x y}=\boldsymbol{\phi} \boldsymbol{R}_{y y}$ where

$$
\begin{aligned}
& \boldsymbol{\gamma}=(\boldsymbol{g}[L], \boldsymbol{g}[L-1], \ldots, \boldsymbol{g}[0]) \\
& \boldsymbol{\phi}=(\boldsymbol{f}[K], \boldsymbol{f}[K-1], \ldots, \boldsymbol{f}[-K])
\end{aligned}
$$

and $\boldsymbol{R}_{x y}$ and $\boldsymbol{R}_{y y}$ are the related block Toeplitz matrices formed using the blocks $\boldsymbol{r}_{x y}[k]$ and $\boldsymbol{r}_{y y}[k]$ respectively. Note that the second order statistics can be estimated by temporal averaging using a sufficiently long training data set because $\boldsymbol{x}$ and $\boldsymbol{y}$ are ergodic random processes. The solution is straightforward: $\phi=\gamma \boldsymbol{R}_{x y} \boldsymbol{R}_{y y}^{-1}$. The minimum value for the equalization error variance is

$$
\mathcal{E}=\operatorname{tr}\left(\boldsymbol{\gamma}\left(\boldsymbol{R}_{x x}-\boldsymbol{R}_{x y} \boldsymbol{R}_{y y}^{-1} \boldsymbol{R}_{y x}\right) \boldsymbol{\gamma}^{*}\right) .
$$

We now consider the target design problem where we minimize the above expression over all causal targets $\boldsymbol{g}$ with lowertriangular $\boldsymbol{g}[0]$ satisfying $\operatorname{det} \boldsymbol{g}[0]=1$. It is easily shown that the solution is given by

$$
\boldsymbol{g}^{*}[0] \gamma / \lambda=(0, \ldots, 0, \boldsymbol{I})\left(\boldsymbol{R}_{x x}-\boldsymbol{R}_{x y} \boldsymbol{R}_{y y}^{-1} \boldsymbol{R}_{y x}\right)^{-1} .
$$

So far we have computed $\boldsymbol{d}[k]:=\boldsymbol{g}^{*}[0] \boldsymbol{g}[k] / \lambda$. We solve for $\boldsymbol{g}$ from $\boldsymbol{d}$ as follows. Pick $\lambda$ to make $\operatorname{det}(\lambda \boldsymbol{d}[0])=1$. Factorize $\lambda \boldsymbol{d}[k]=\boldsymbol{g}^{*}[0] \boldsymbol{g}[0]$ so that $\boldsymbol{g}[0]$ is lower-triangular and solve for the remaining terms: $\boldsymbol{g}[k]=\lambda\left(\boldsymbol{g}^{*}[0]\right)^{-1} \boldsymbol{d}[k]$.

\section{Application to Multi-track Recording}

We illustrate a simple application of this work to a multitrack recording system [2], [3] consisting of $N=2$ adjacent tracks and 2 read-heads that simultaneously read the data bits from the tracks. The readback signals are affected by ISI (within a track) and inter-track interference (ITI) and additive white Gaussian noise. After analog-filtering and Nyquist sampling, the channel is modeled as a discrete-time $2 \times 2$ MIMO ISI channel (4) where $\boldsymbol{w}$ is white Gaussian noise with $\boldsymbol{S}_{w}(\omega)=\sigma_{w}^{2} \boldsymbol{I}$ and $\boldsymbol{x} \in\{-1,+1\}^{2}$. The channel response is

$$
\boldsymbol{h}[n]=\left(\begin{array}{cc}
1 & \mu_{1} \\
\mu_{2} & 1
\end{array}\right) h_{0}(t)
$$

where $\mu_{1}$ and $\mu_{2}$ are the intensities of ITI from one track to the other, $h_{0}(t)=h_{T}(t)-h_{T}(t-1)$ is the bit response in the absence of ITI corresponding to the perpendicular recording transition response $h_{T}(t)=\operatorname{erf}(2 n \sqrt{\ln 2} / D)$ at normalized recording density $D$. We adopt the signal-to-noise ratio (SNR) definition in [13]: $\mathrm{SNR}=2 D \sqrt{\ln 4 / \pi} / \sigma_{w}^{2}$.

In Fig. 3, we compare the performance of different equalizer/target pairs designed using 3 constraints: identity tap constraint, orthonormality constraint [11], and monic determinant constraint. The targets and equalizers are $2 \times 2$ MIMO FIR filters with 3 taps (causal) and 15 taps (origin centered) respectively. We assume $\mu_{1}=0.2, \mu_{2}=0.3$ and $D=2$. Although the performance of the identity-tap constraint matches that of

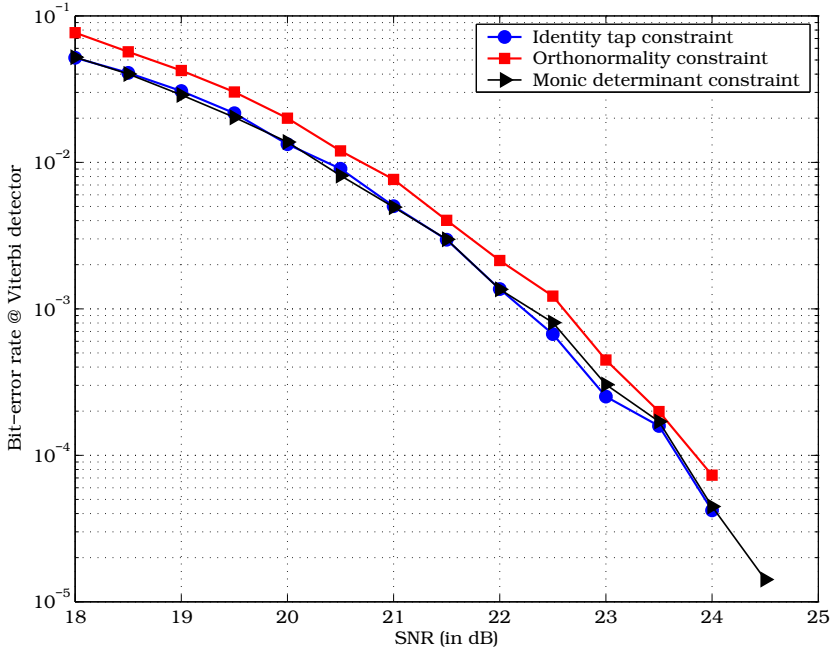

Fig. 3. Performance of multi-track multi-head systems with 3 different constraints for equalizer and target filters design

the monic determinant constraint, it incurs a slightly higher decoding cost because the branch metric is adjusted for the non-identity form of the equalization error covariance matrix. The design based on the orthonormality constraint leaves the estimation error colored and its performance is worse than that of the other two constraints.

\section{SUMMARY}

We considered the problem of reduced complexity detection for MIMO ISI channels with additive colored noise. Using the canonical noise whitened form of the channel, we first presented expressions for the optimal MAP based sequence or symbol detection. We then studied the use of channel shortening equalization for to reduce complexity of the detector. In doing so, we generalized the notion of a posteriori equivalence and derived the family of optimal MIMO equalizer and targets. These solutions are shown to be releted to the MIMO DFE designed with a monic determinant target constraint. These results are applicable to a wide class of MIMO ISI channels such as multi-track recording systems and MIMO wireless channels.

\section{REFERENCES}

[1] I. E. Telatar, "Capacity of multi-antenna Gaussian channels," AT\&T Bell Labs Technical Memorandum.

[2] E. Soljanin and C. N. Georghiades, "Multihead detection for multitrack recording channels," IEEE Trans. on Information Theory, vol. 44, no. 7, pp. 2988-97, 1998.

[3] E. Kurtas, J. G. Proakis, and M. Salehi, "Reduced complexity maximum likelihood sequence estimation for multitrack high-density magnetic recording channels," IEEE Trans. on Magnetics, vol. 35, no. 4, pp. 218793, 1999.

[4] R. Venkataramani and M. F. Erden, "A Posteriori Equivalence: A New Perspective for Design of Optimal Channel Shortening Equalizers," ArXiv e-prints, vol. 710, Oct. 2007, (submitted to IEEE Trans. on Information Theory).

[5] R. Venkataramani and M. F. Erden, "A family of equalizers for optimal sequence detection," in Proc. Int. Conf. Acoustics and Speech Signal Proc., Toulouse, France, May 2006. 
[6] D. C. Youla and N. N. Kazanjian, "Bauer-type factorization of positive matrices and the theory of matrix polynomials orthogonal on the unit circle," IEEE Trans. on Circuits and Systems, vol. 25, no. 2, pp. 57-69, February 1978.

[7] A. H. Sayeed and T. Kailath, "A survey of spectral factorization methods," Numerical Linear Algebra with Applications, vol. 8, no. 6-7, pp. 467-496, 2001.

[8] J. M. Cioffi, G. P. Dudevoir, M. V. Eyuboglu, and G. D. Forney, "MMSE decision feedback equalizers and coding-part I: Equalization results," IEEE Trans. Inform. Theory, vol. 43, no. 10, pp. 2582-2594, October 1995.

[9] — - "MMSE decision feedback equalizers and coding-part II: Coding results," IEEE Trans. Inform. Theory, vol. 43, no. 10, pp. 2595-2604, October 1995.

[10] R. F. H. Fischer, "Sorted spectral factorization of matrix polynomials in MIMO communications," IEEE Trans. on Communications, vol. 53, no. 6, June 2005.

[11] N. Al-Dhahir, "FIR channel shortening equalizers for MIMO ISI channels," IEEE Trans. on Communications, vol. 49, no. 2, pp. 213-218, Feb. 2001.

[12] — "Finite-length multi-input multi-output MMSE-DFE," IEEE Trans. on Signl Processing, vol. 48, no. 10, pp. 29213-36, Oct. 2000.

[13] B. Vasic and E. Kurtas, Eds., Coding and Signal Processing for Magnetic Recording Systems. CRC Press, 2005. 